\title{
Avaliação do autoconhecimento de fatores de risco cardiovascular segundo os grupos socio-organizacionais urbano e rural de um município do Rio de Janeiro
}

\section{Self-knowledge assessment of cardiovascular risk factors according to urban and rural socio- organizational groups in a Rio de Janeiro city}

Raul Ferreira de Souza Machado ${ }^{\dagger^{*}}$, Caio Teixeira dos Santos ${ }^{\dagger}$, João Paulo Brum Paes ${ }^{\dagger}$, Sara Cristine Marques dos Santos ${ }^{\dagger}$, Thaís Lemos de Souza Macêdo ${ }^{\dagger}$, Ivana Picone Borges de Aragão

Como citar esse artigo.Machado, R. F. S; Dos Santos, C. T; Paes, J. P. B; Dos Santos, S. C. M; Macedo, T. L. S; De Aragão, I. P. B. Avaliação do autoconhecimento de fatores de risco cardiovascular segundo os grupos socioorganizacionais urbano e rural de um município do rio de janeiro. Revista de Saúde. 2020 Jul./Dez.; 11 (2): 02 - 09.

\section{Resumo}

O aumento da expectativa de vida mundial e ocidentalização contribuíram para o aumento da prevalência de doenças cardiovasculares (DCV) e, diferentes dinâmicas entre áreas rurais e urbanas, podem ter impacto na prevenção primária. A prevalência e autoconhecimento dos fatores de risco (FR) para DCV nas populações de zona rural (ZR) e zona urbana periférica (ZUP) de uma cidade brasileira foram avaliados e comparados. Estudo observacional e transversal entre 2017-2019 com aplicação de questionário anônimo. Total 291 indivíduos, 88 na ZR e 203 na ZUP sendo variáveis avaliadas, respectivamente: média idade 56 e 45 anos; $59 \%$ e $64 \%$ mulheres; $23,9 \%$ e $20,7 \%$ ex-fumantes; $13,6 \%$ e $20,7 \%$ fumantes; $51 \%$ e 39,9\% afirmaram PA>120/80mmHg com $17 \%$ e 23,6\% afirmaram hipertensão; 78,4\% e 75,4\% mediram colesterolemia; $26,1 \%$ e $15,8 \%$ relataram hipercolesterolemia, medicados em $21,6 \%$ e $8,4 \% ; 13,6 \%$ e $14,2 \%$ glicemia $\geq 126 \mathrm{mg} / \mathrm{dL}$ ou diabetes mellitus; média de IMC 28 e 27 ; história familiar parente primeiro grau masculino $25 \%$ e $15,8 \%$ e feminino $15,9 \%$ e $18,2 \% ; 3,4 \%$ e $10,3 \%$ IAM prévio; $43,2 \%$ e $56,7 \%$ cansaço; $19,3 \%$ e $36,9 \%$ palpitação; $26,1 \%$ e $34,8 \%$ dispneia; $11,4 \%$ e $13,8 \%$ desmaio; $44,31 \%$ e $47,8 \%$ claudicação; $19,3 \%$ e $20,7 \%$ dor torácica aos esforços e repouso $11,4 \%$ e $13,8 \% ; 27,3 \%$ e $23,1 \%$ acompanhamento com cardiologista; estresse muito frequente $22,7 \%$ e $36,9 \%$. Evidente prevalência e desconhecimento de FR em ambos os grupos, sendo mais evidentes na ZR, apesar do histórico pessoal e sintomas terem sido mais evidentes na ZUP. A prevenção primária deve ser permanentemente incentivada em ambas as comunidades.

Palavras-chave: Doenças Cardiovasculares; Prevenção Primária; Fatores De Risco; Área Urbana; Zona Rural. $14.2 \%$ blood glucose $\geq 126 \mathrm{mg} / \mathrm{dL}$ or diabetes mellitus; mean BMI 28 and 27; family history first-degree relative male $25 \%$ and $15.8 \%$ and female $15.9 \%$ and $18.2 \% ; 3.4 \%$ and $10.3 \%$ previous AMI; $43.2 \%$ and $56.7 \%$ tiredness; $19.3 \%$ and $36.9 \%$ palpitation; $26.1 \%$ and $34.8 \%$ dyspnea; $11.4 \%$ and $13.8 \%$ faint; $44.31 \%$ and $47.8 \%$ lameness; $19.3 \%$ and $20.7 \%$ chest pain on exertion and rest $11.4 \%$ and $13.8 \% ; 27.3 \%$ and $23.1 \%$ follow-up with a cardiologist; very frequent stress $22.7 \%$ and $36.9 \%$. Evident prevalence and ignorance of RF in both groups, being more evident in the ZR, despite the personal history and symptoms were more evident in the ZUP. Primary prevention must be permanently encouraged in both communities.

Keywords: Cardiovascular Diseases; Primary Prevention; Risk Factors; Urban Area; Countryside.

\section{Introdução}

$\mathrm{O}$ aumento da expectativa de vida mundial e a ocidentalização de hábitos e estilo de vida corroboraram para o aumento da prevalência de doenças cardiovasculares (DCV). Estima-se o valor de 17,7 milhões de óbitos por doenças cardiovasculares (DCV), 31\% das mortes em nível global. Só no Brasil, em uma década, houve 3,5 milhões ${ }^{1}$. Com o advento das variadas terapias e o aumento da expectativa de vida, os índices têm se mostrado crescentes, sobretudo os referentes a hipertensão arterial sistêmica (HAS) e insuficiência cardíaca, sendo essa última representante de um processo final da maioria das doenças cardíacas. Além disso, as condições existentes em áreas urbanas podem ter impacto na saúde, relacionadas ao contexto social e ambiental, à promoção da saúde e à atenção 
primária².

Dentre as DCV, as cardiopatias isquêmicas representam uma parcela importante das estatísticas, sendo as principais relacionadas ao avançar da idade, sedentarismo, obesidade, estresse, tabagismo, dislipidemia, hipertensão e diabetes mellitus (DM), que constituem fatores de risco (FR) intimamente ligados aos hábitos de vida da população. Está associada a queda precoce e significativa da qualidade de vida de seus portadores e a importante morbidade, com um impacto econômico expressivo, visto a necessidade de cuidados contínuos e prolongados e reabilitação, além do custo indireto representado por improdutividade e morte prematura ${ }^{3}$.

A aterosclerose, principal fator na patogênese das cardiopatias isquêmicas, deixou de representar uma doença crônico-degenerativa exclusiva de pacientes de idade avançada, sendo considerada como doença inflamatória crônica subclínica, presente já na infância. Diversos dos fatores desencadeantes da formação das placas ateroscleróticas nas artérias coronárias são mutáveis e, se identificados precocemente e tratados, capazes de reduzir de forma significativa os crescentes índices apresentados e, consequentemente, os custos relacionados e as diversas comorbidades associadas ${ }^{4}$.

Concretizando um esforço mundial, a redução do risco de morte prematura por DCV foi definida como uma das principais prioridades de desenvolvimento sustentável das Organizações das Nações Unidas (ONU) até 20305. A Organização Mundial da Saúde (OMS) define os componentes sociais da saúde como as "circunstâncias em que os indivíduos nascem, crescem, vivem, trabalham e envelhecem, além dos sistemas empregados para lidar com doenças". Nesse cenário, a associação dos fatores sociais com as DCV, embora ainda pouco estudada, justifica sua importância ${ }^{6}$.

É visto na literatura uma relação negativa entre a mortalidade cardiovascular (CV) e a distância entre a residência dos pacientes e os centros de saúde, indicando que os pacientes que residem nas periferias têm maiores taxas de complicações $\mathrm{CV}^{7}$. A faixa etária e a autopercepção de saúde estão associadas a DCV, como hipertensão arterial sistêmica, insuficiência cardíaca, acidente vascular encefálico e infarto agudo do miocárdio ${ }^{2}$. Em estudo brasileiro, as barreiras geográficas da zona rural constituíram fator impeditivo para o maior acesso aos serviços de saúde dessa população ${ }^{8}$, enquanto a dificuldade de locomoção se mostrou como fator limitante ao acesso entre a população urbana'.

A literatura também sugere diferença estatística no que tange à adesão a tratamento medicamentoso entre os indivíduos de zona rural e de zona urbana ${ }^{9}$, sendo esta a maior entre os de zona rural, o que se expressa como um fator importante, visto que a não adesão ao tratamento medicamentoso reduz os resultados terapêuticos, sobretudo em doenças crônicas.

Mundialmente, incluindo o Brasil, houve evidência que o baixo nível socioeconômico está relacionado ao aumento da mortalidade por DCV e por todas as causas. O trabalho com empregos de baixa remuneração e consequente baixa renda, baixo nível educacional e locais pobres residenciais definem um nível socioeconômico baixo. Tendo em vista essa associação com impacto na mortalidade $\mathrm{CV}$, atualmente foi considerado como fator de risco $\mathrm{CV}$ segundo as diretrizes brasileiras de prevenção $\mathrm{CV}^{10}$.

A importância da educação vem sendo fortemente relacionada à mortalidade $\mathrm{CV}$, independentemente de outros fatores sociais ${ }^{11}$. Um estudo epidemiológico demonstrou a relação da redução da mortalidade CV com progressão dos indicadores da macroeconomia como o Produto Interno Bruto per capita (PIBpc) dos diversos municípios do Estado nas últimas décadas ${ }^{12}$.

Importantemente, devem ser consideradas as emissões de gases na atmosfera terrestre no contexto urbano através das indústrias, veículos auto motorizados como problema em relação ao impacto na saúde $\mathrm{CV}$, assim como considerar a ocorrência de queimadas de biomassa nesse contexto em ambiente rura ${ }^{13}$.

O objetivo do presente estudo foi avaliar a prevalência e o autoconhecimento dos FR para DCV nas populações residentes em zona rural (ZR) e zona urbana periférica (ZUP) do município de Vassouras, localizado no Estado do Rio de Janeiro/Brasil e comparar as variáveis estudadas no que tange $\mathrm{FR} \mathrm{CV}$ entre as duas populações.

\section{Métodos}

Foi realizado um estudo observacional e transversal entre março de 2017 e abril de 2019, através da aplicação e validação de questionário, adaptado de modelo pré-existente, de forma anônima, acerca do autoconhecimento dos FR para CV em moradores de bairros socioeconomicamente desfavorecidos tanto em zona rural, como urbana da periferia do município de Vassouras, Rio de Janeiro.

Este artigo faz parte do projeto de pesquisa intitulado "Rastreamento do Autoconhecimento dos Fatores de Risco Cardiovascular em População da Periferia de Vassouras", aprovado pelo CEP (Comitê de Ética em Pesquisa) sob parecer de número 1.883.015.

Critério de inclusão: ser morador da periferia do município de Vassouras na zona urbana ou rural; ambos os sexos; maiores de 20 anos de idade, idade estabelecida a partir dos dados obtidos no Departamento de Informática do sistema único de Saúde (DATASUS).

Os moradores do município de Vassouras foram divididos em dois grupos: zona urbana periférica - 
ZUP, que incluiu os moradores dos bairros de Itakamosi e Ipiranga; zona rural - ZR, que incluiu os moradores do bairro de Massarambá. Os questionários de ambos os grupos foram comparados entre si.

$\mathrm{O}$ número total de moradores foi identificado e a seguir procedida a aplicação do questionário anônimo contendo 23 perguntas de respostas rápidas, em relação a autoconhecimento dos FR para $\mathrm{CV}$, mencionadas a seguir: idade; tabagismo ( $\operatorname{sim}$ ou não); pressão arterial $>$ 120/80 mmHg (sim ou não, desconhecimento); dislipidemia e valor do colesterol total, LDL e HDL (sim, não, desconhecimento); história familiar de doença arterial coronariana (DAC) ou acidente vascular cerebral em parentes de primeiro grau (sim, não, desconhecimento); DM e valor da glicemia $\geq 126 \mathrm{mg} / \mathrm{dl}$ (sim, não, desconhecimento); valor do índice de massa corporal (IMC), peso e altura (valor, desconhecimento); prática de exercícios físicos maior que 150 minutos por semana (sim, não).

O questionário incluiu também as seguintes perguntas: histórico de infarto ou angina pectoris (sim, não, desconhecimento); sintomas de fadiga ou palpitações ou falta de ar ou desmaio sem explicação ou dor nas pernas ao caminhar, desconforto no peito, pescoço, mandíbulas ou ombro com esforço ou repouso (sim, não), sendo considerado aquelas com 2 ou mais sintomas; prevenção cardiológica regularmente (sim, não); auto percepção do estresse (nunca, raramente, pouco frequente, frequentemente, muito frequente); local de moradia (zona urbana ou zona rural).

As variáveis analisadas correspondem a cada resposta das perguntas contidas no questionário que foram inseridas em um banco de dados no programa Excel. Os dados foram analisados através da utilização de porcentagens e variáveis categóricas através de Figuras e tabelas, e médias para as variáveis contínuas. Cada questionário foi analisado separadamente para obter-se uma análise descritiva dos dados.

Cada variável foi comparada entre os dois grupos estudados (ZUP e ZR), a fim de identificar relação entre a região de moradia, rural ou urbana, e o autoconhecimento de FR para DCV.

\section{Resultados}

Total de 291 indivíduos foram estudados, sendo $88(30,2 \%)$ moradores ZR e $203(69,8 \%)$ de ZUP. De acordo com as respostas obtidas no questionário, identificou-se, respectivamente nos grupos ZR e ZUP: média de idade foi de 56 e 45 anos; sexo feminino em $52(59 \%)$ e $130(64 \%)$; afirmaram ser tabagistas (figura 1) em $12(13,6 \%)$ e $42(20,7 \%)$; ex-tabagistas em 21 $(23,9 \%)$ e $42(20,7 \%)$; negaram tabagismo durante a vida em $55(62,5 \%)$ e $119(58,6 \%)$.

Em relação aos níveis tensionais, respectivamente,

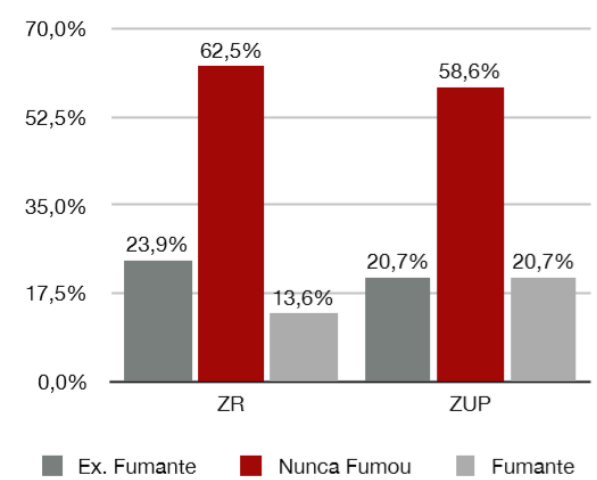

Figura 1. : Porcentagem de indivíduos fumantes, exfumantes e que nunca fumaram por zona (rural ou urbana periférica).

os grupos ZR e ZUP informaram: negaram medidas tensionais $>120 / 80 \mathrm{mmHg}$ em $37(42 \%)$ e 109(53,7\%); afirmaram valores acima da medida $>120 / 80 \mathrm{mmHg}$ em $45(51 \%)$ e $81(39,9 \%)$; porém houve moradores que desconheciam sua condição ou não informaram em $6(6,81 \%)$ e $13(6,40 \%)$; afirmaram conhecimento sobre os próprios valores da pressão arterial como compatíveis com hipertensão arterial (figura 2) em 15 $(17 \%)$ e 48 (23,6\%); como pré-hipertensão em $5(5,7 \%)$ e $24(11,8 \%)$; como não hipertensos em $17(19,3 \%)$ e $92(45,3 \%)$ na ZUP; não souberam informar os níveis tensionais em $18(20,45 \%)$ e $43(21,18 \%)$.

Um total de $231(79,38 \%)$ indivíduos souberam informar os valores da medida da pressão arterial, 71 $(80,68 \%)$ da ZR e $160(78,81 \%)$ da ZUP. As médias dos valores informados para o grupo total, ZR e ZUP foram, respectivamente: $127,49 \times 81,30 \mathrm{mmHg}$ ,129,57x81,43mmHg , 126,57x81,25mmHg.

Quando perguntados a respeito dos níveis sanguíneos de colesterol total, os grupos ZR e ZUP responderam, respectivamente: negaram realização de exame de sangue para verificação dos níveis de colesterolemia total em $14(15,9 \%)$ e $45(22,1 \%)$;

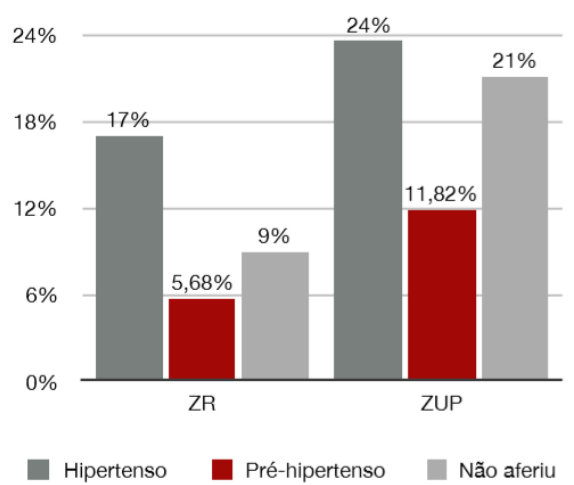

Figura 2: Porcentagem de indivíduos sabidamente hipertensos por zona (rural ou urbana periférica). 
afirmaram já terem realizado o exame em $69(78,4 \%)$ e 153 (75,4\%); não informaram ou desconheciam o seu valor em $5(5,7 \%)$ e $2(0,98 \%)$; afirmaram colesterolemia $>200 \mathrm{mg} / \mathrm{dl}$ (figura 3 ) em $23(26,1 \%$ ) e 32 $(15,8 \%)$; negaram valores de colestorolemia $>200 \mathrm{mg} /$ dl em $25(28,4 \%)$ e 94 (46,3\%); afirmaram níveis de HDL colesterolemia $<45 \mathrm{mg} /$ dl (figura 4 ) em $8(9 \%)$ e 20 (9,8\%); negaram HDL $<45 \mathrm{mg} / \mathrm{dl}$ em $15(17 \%)$ e 26 $(12,80 \%)$; negaram uso de medicamentos para controle do colesterol em $58(65,9 \%)$ e $153(75,4 \%)$; relataram uso da medicação em $19(21,6 \%)$ e $17(8,4 \%)$.

Um total de $56(19,24 \%)$ indivíduos souberam informar os valores de colesterolemia total e $33(11,34 \%)$ da fração HDL. A média dos valores para colesterolemia total e fração HDL informados, respectivamente, para os grupos total, ZR e ZUP de 201 e $50 \mathrm{mg} / \mathrm{dl}, 237,5$ e $46 \mathrm{mg} / \mathrm{dl}, 184,5$ e $52,2 \mathrm{mg} / \mathrm{dl}$.

Sobre os índices glicêmicos e medida antropométrica, os grupos ZR e ZUP responderam, respectivamente: afirmaram glicemia $\geq 126 \mathrm{mg} / \mathrm{dl}$ ou DM (figura 5) em 12 (13,6\%) e 29 (14,3\%); afirmaram índice de massa corporal $\geq 25$ (figura 6) em 15 (17\%) e

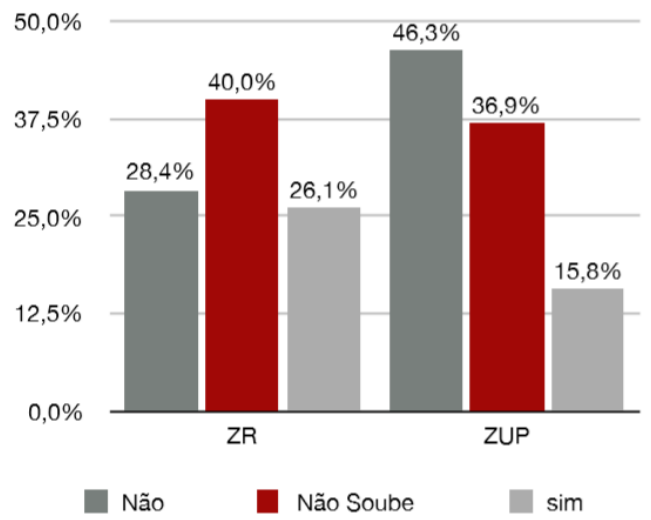

Figura 3: Porcentagem de indivíduos com colesterolemia total sabidamente maior que $200 \mathrm{mg} / \mathrm{dl}$ por zona (rural ou urbana periférica).

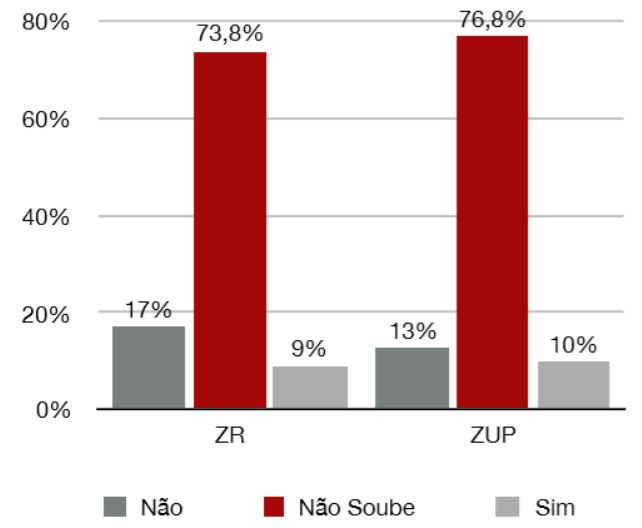

Figura 4: Porcentagem de indivíduos com HDL colesterol sabidamente menor que $45 \mathrm{mg} / \mathrm{dl}$ por zona (rural ou urbana periférica).

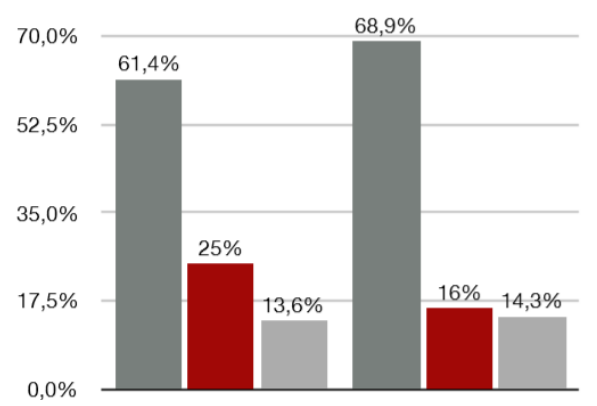

Figura 5: Porcentagem de indivíduos sabidamente diabéticos ou com glicemia em jejum maior ou igual que 126 $\mathrm{mg} / \mathrm{dl}$ por zona (rural ou urbana periférica).

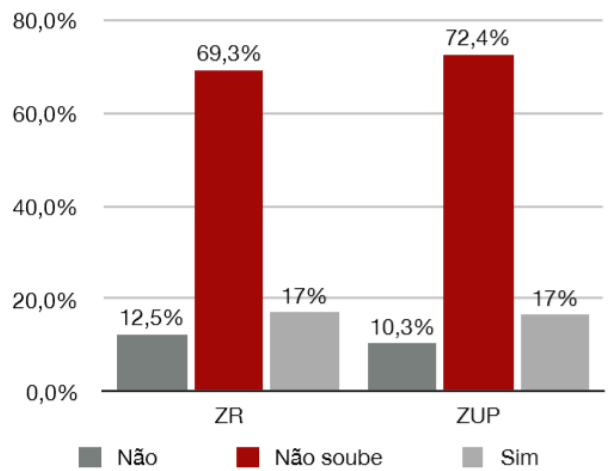

Figura 6: Porcentagem de indivíduos que afirmaram índice de massa corporal maior ou igual que 25 por zona (rural ou urbana periférica).

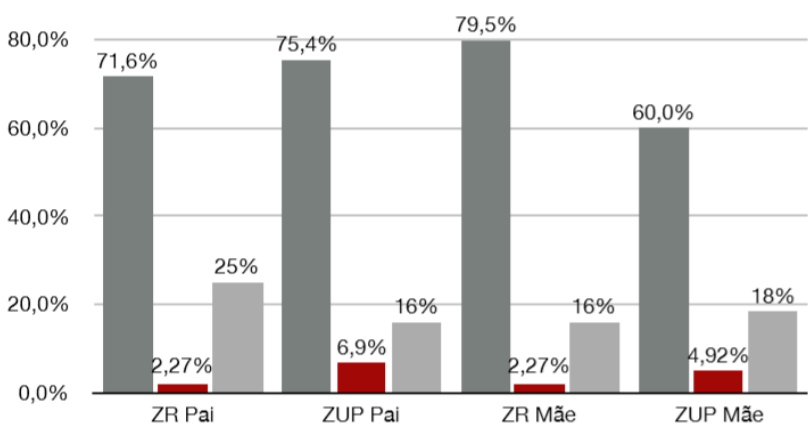

Figura 7: Porcentagem de indivíduos com história familiar de infarto agudo do miocárdio em pai ou mãe por zona (rural ou urbana periférica).

34 (16,7\%); não souberam informar em 62 (70,45\%) e $147(72,41 \%)$.

Os indivíduos foram questionados sobre peso e altura para posterior cálculo do IMC. Dos grupos ZR e ZUP, souberam responder, respectivamente: seu peso $70(79,54 \%)$ e $181(89,16 \%)$; sua altura $61(69,32 \%)$ e $161(79,31 \%)$. As médias de IMC calculadas para o grupo total, ZR e ZUP foram, respectivamente de 27,5, 28 e 27.

Em relação à história familiar(figura 7) e pregressa (figura 8) para infarto agudo do miocárdio (IAM) os grupos ZR e ZUP responderam, respectivamente: pai já 
sofreu IAM em $22(25 \%)$ e 32 (15,8\%); negaram em $63(71,6 \%)$ e $153(75,4 \%)$; mãe já sofreu IAM em 70 $(79,5 \%)$ e $122(60,1 \%)$; referiram passado de IAM em $3(3,41 \%)$ e $21(10,35 \%)$; negaram passado de IAM em $82(93,18 \%)$ e $179(88,17 \%)$ e não souberam responder em $3(3,41 \%)$ e $3(1,48 \%)$.

Em relação aos sintomas observados pelos entrevistados (figura 9), os grupos ZR e ZUP responderam, respectivamente: afirmaram cansaço em $38(43,2 \%)$ e $115(56,7 \%)$; palpitações em $17(19,3 \%)$ e 75 (36,9\%); falta de ar em $23(26,1 \%)$ e $71(34,8 \%)$; desmaios sem explicação em $10(11,4 \%)$ e $28(13,8 \%)$; percepção de dor nas pernas ao caminhar em 39 $(44,31 \%)$ e $97(47,8 \%)$; dor no peito aos esforços em 17 $(19,3 \%)$ e $42(20,7 \%)$; dor no peito em repouso em 10 $(11,4 \%)$ e $28(13,8 \%)$.

No que condiz ao acompanhamento médico, respectivamente, os grupos da ZR e ZUP demonstraram a seguinte prática: consultas regulares ao cardiologista $24(27,3 \%)$ e $47(23,1 \%)$ com relato da média em anos de frequência as consultas de 18,5 e 7 anos.

Em relação à autopercepção do estresse (figura 10 ), os grupos ZR e ZUP informaram respectivamente: sentiam às vezes em $25(28,4 \%)$ e $61(30 \%)$; muito frequente em $20(22,7 \%)$ e $75(36,9 \%)$; nunca em 25 $(28,4 \%)$ e $25(12,3 \%)$; pouco frequente em $8(9,1 \%)$ e $17(8,4 \%)$; quase nunca em $10(11,4 \%)$ e $25(12,3 \%)$.

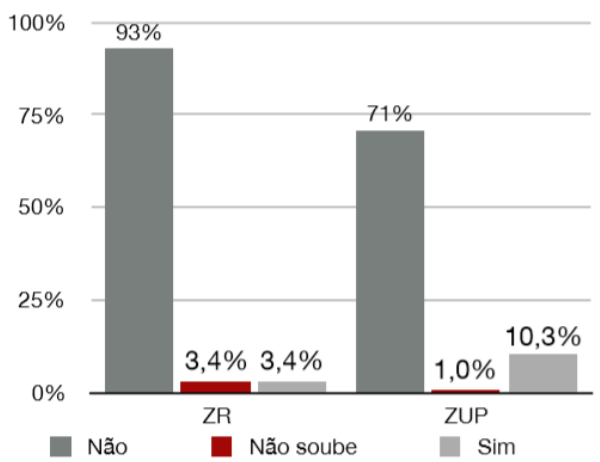

Figura 8: Porcentagem de indivíduos com história pregressa de infarto agudo do miocárdio por zona (rural ou urbana periférica).

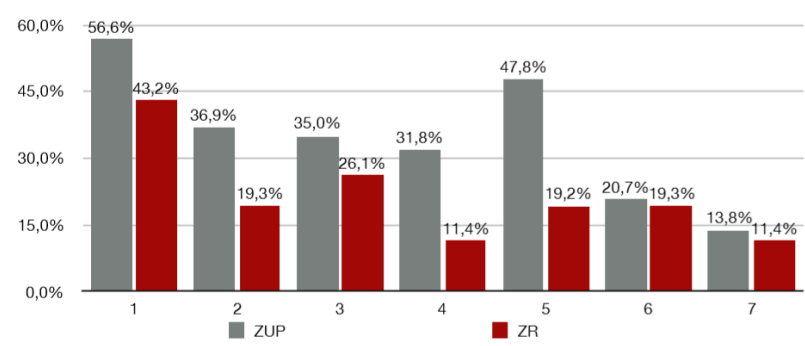

Figura 9: Porcentagem de indivíduos que relataram já ter apresentado os seguintes sintomas: 1) cansaço; 2) palpitação; 3) falta de ar; 4) desmaio sem explicação; 5) dor nas pernas ao caminhar; 6) dor no peito aos esforços; 7) dor no peito em repouso, por zona (rural ou urbana periférica).

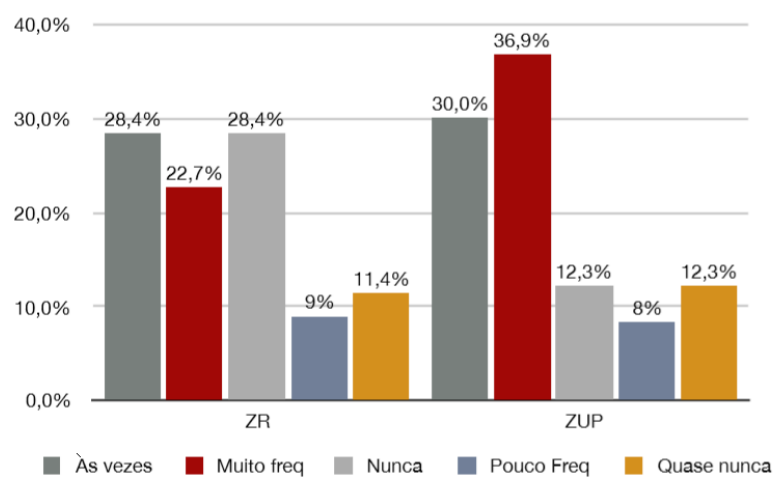

Figura 10: Autopercepção do estresse em indivíduos das zonas rural e urbana periférica (\%).

\section{Discussão}

A desigualdade socioeconômica na população brasileira contribuiu para diferentes frequências de doenças crônicas, demonstrando valores mais elevados nos segmentos economicamente desfavorecidos ${ }^{14}$, da mesma forma que esse estudo pode observar em relação a frequência nas autodeclarações de hipertensão, diabetes, dislipidemia, história de doença coronariana em ambos os grupos, independentemente das diferentes prevalências entre eles.

De acordo com a literatura, cerca 75 a $80 \%$ dos pacientes acometidos por DAC possuem FR convencionais ou clássicos em sua história clínica, representados por HAS, tabagismo, hipercolesterolemia, DM, idade avançada, sexo masculino e antecedentes familiares, além de sedentarismo, estresse emocional e obesidade $^{15,16,17,18}$.

A crescente urbanização visível em todo o mundo trouxe consigo mudanças de hábitos e estilo de vida que corroboraram para o aumento da prevalência dos FR na população, a exemplo da adoção de hábitos alimentares inadequados e sedentarismo, que favorecem ao crescimento dos níveis de obesidade, além do estresse mental ou emocional, que é um dos maiores problemas das sociedades modernas e também tem importância neste aumento, visto que o sistema CV é afetado ${ }^{19}$.

$\mathrm{O}$ presente estudo observou que havia maior porcentagem de respostas positivas para os níveis de pressão arterial, colesterol, glicemia, IMC e estresse na população periférica urbana, corroborando para os achados da literatura.

Diversos estudos evidenciam a relação do envelhecimento da população com a presença de doenças cardiovasculares, além do fato do correto tratamento dessas condições permitir o aumento da longevidade e maior exposição aos fatores de risco ${ }^{20}$.

Além das condições físicas e individuais, características sociodemográficas têm sido relacionadas ao desenvolvimento de DCV, sendo os FR mais 
frequentes em populações com menor poder econômico

e cultural ${ }^{21}$. Nesse contexto, é importante conhecer a realidade local e as características de cada grupo populacional ao se avaliar risco $\mathrm{CV}$, de modo a buscar por ações factíveis para cada grupo, de acordo com a estrutura física, acesso à saúde e organização social.

Os FR ambientais, comportamentais e ocupacionais apresentam forte relação com as causas de mortalidade e, por isso, requerem avaliações concomitantes aos FR clássicos, visto que até estes podem ser influenciados pela complexa relação entre o indivíduo e o contexto em que este vive. A íntima associação entre as condições socioeconômicas das populações e suas condições de saúde tem sido cada vez mais abordada: diversos estudos prospectivos mostram que o baixo nível educacional, a baixa renda, empregos de baixo status ou a moradia em áreas mais pobres são determinantes sociais vistos como fatores de risco independentes para doenças, contribuindo para o aumento de todas as causas de morte ${ }^{2,22}$.

A associação entre as condições socioeconômicas e a mortalidade por DAC (RR entre 1,3 e 2) pode ser bem visualizada quando foram comparadas as taxas de mortalidade por DAC ao redor do mundo com os cenários econômicos, sociais e de desenvolvimento de cada local ${ }^{23,24}$. Nos países desenvolvidos, a queda da mortalidade foi associada ao fim da Segunda Guerra Mundial. Em países em desenvolvimento como o Brasil, esta queda se expressou em períodos de maior crescimento econômico, melhorias educacionais, de infraestrutura e sanitárias, com redução de doenças infecciosas, principalmente nas fases iniciais da vida, o que pode estar relacionado à diminuição da mortalidade CV na fase adulta ${ }^{10}$.

Hoje, já se conhece que o aumento do Índice de Desenvolvimento Humano (IDH), da escolaridade do produto interno bruto (PIB) per capita e a queda da mortalidade infantil são fatores que aumentam a sobrevida por $\mathrm{DAC}^{10}$. Afora isto, características muito comumente relacionadas ao meio urbano, sobretudo às periferias, são tratadas como fatores capazes de limitar a promoção da saúde, como: aglomeração populacional; altos índices de violência; poluição ambiental, sobretudo atmosférica; saneamento básico e acesso à água potável precários ou ausentes; e alto nível de ruídos ${ }^{25}$.

Isto poderia explicar o porquê de, embora os moradores de zona rural apresentem, em vários aspectos, menor conhecimento acerca de cuidados cientificamente respaldados com a saúde, moradores de ZUP costumarem ser mais expostos a más condições de vida e estresse, condições que ampliam o número e a presença de FR para doenças crônicas não transmissíveis nessas populações, além de menor escolaridade ${ }^{26} . \mathrm{Na}$ população que foi avaliada nesse estudo em relação a autopercepção do estresse, $36,94 \%$ dos moradores de ZUP relataram senti-lo com muita frequência, enquanto apenas $22,73 \%$ alegaram o mesmo no grupo de ZR.

Estudos sugerem que a escolaridade é menor nos grupos populacionais rurais que urbanos, de modo que os indivíduos de ZR estariam mais expostos a fatores prejudiciais à saúde, por menor capacidade de compreensão sobre eles. Todavia, um estudo comparativo com idosos portadores de DM moradores de zonas rurais e urbanas trouxe dados de escolaridade que divergentes, sugerindo menor escolaridade em indivíduos de zonas urbanas periféricas, com a zona rural apresentando maior percentual de indivíduos estudados com quatro a oito anos de estudo $(44,2 \%)$, enquanto a zona urbana apresentava seu maior percentual na faixa de um a quatro anos $(41,1 \%)$, apontando para uma maior escolaridade entre os indivíduos de área rural ${ }^{27}$.

No presente estudo, foi observado que a ausência de conhecimento sobre a saúde foi maior entre os moradores de ZR, quando questionados acerca de suas condições, pois $51,14 \%$ não soube informar seus valores pressóricos (contra 39,9\% da ZUP), 4,54\% não souberam informar se já haviam realizado dosagem de colesterol (contra $0,98 \%$ da ZUP) e 3,41\% não souberam informar se já haviam realizado exame de glicemia (contra $0,98 \%$ da ZUP).

Os testes laboratoriais para avaliação dos níveis de glicemia estão entre os rotineiros na população em geral, no presente estudo, entre aqueles que alegaram já ter realizado o exame, $25 \%$ dos indivíduos da ZR desconheciam seus valores de glicemia, contra $16,36 \%$ entre os moradores da ZUP.

Um estudo cujo objetivo foi avaliar a validade do diabetes auto-referido mostrou que a escolaridade e a utilização dos serviços de saúde, facilitada pela presença de atendimento de fácil acesso à população, foram os principais determinantes da capacidade do indivíduo de informar de forma correta sobre sua condição de saúde lançando luz, mais uma vez, para os determinantes sociais da saúde ${ }^{28}$.

Estudo comparativo realizados em indivíduos idosos das duas diferentes zonas permitiu observar que o relato de algumas morbidades foi estatisticamente diferente entre elas. Condições psiquiátricas como depressão, ansiedade, cefaleia e Doença de Alzheimer foram mais prevalentes em zona urbana, além de sintomas como palpitação e insônia. Quanto ao DM, o cenário foi oposto: apresentou maior prevalência entre os moradores da zona rural do que nos da zona urbana $(13,3 \%$ vs $4,6 \% ; p=0,018)$, sendo este fator de risco isolado para $\mathrm{DCV}^{27}$.

Além dos FR clássicos, indivíduos de zona rural também apresentam alguns adicionais, como a exposição a agrotóxicos e pesticidas, que apresenta associação com diversas alterações orgânicas, embora um estudo de 2011 não tenha registrado associação entre relato de DCV com contato com agrotóxicos. ${ }^{29}$

Em conformidade com dados previamente obtidos 
em estudos realizados com outros grupos populacionais na literatura, foi possível evidenciar que houve maior prevalência de tabagismo em moradores da zona urbana periférica $(20,69 \%$ ZUP versus $13,64 \% \mathrm{ZR})$, assim como o DM (14, $28 \%$ ZUP versus $13,63 \%$ de ZR).

$\mathrm{O}$ sobrepeso foi mais evidente em indivíduos moradores da zona rural $(17,04 \%$ contra $16,74 \%$ de ZUP), embora deva ser ressaltado que dois terços da população não forneceram dados suficientes para $\mathrm{o}$ cálculo do IMC, o que impediu de se estabelecer um padrão entre os locais de residência.

A presença de fatores de risco cardiovasculares modificáveis, conforme visto na amostra, também vai ao encontro da literatura, sendo um campo de intervenção essencial em medidas mitigadoras da morbimortalidade por $\mathrm{DCV}^{14}$.

Grande parte da população estudada relatou ser portadora de FR, porém demonstrando a falta de conhecimento ao responderem sobre os valores de pressão arterial, glicemia e colesterolemia, além da presença de sintomas sugestivos do sistema CV. É válido dar ênfase à atenção primária com equipes de saúdes locais e ressaltar sua grande capacidade de captar e manejar os fatores de risco, auxiliando na melhoria dos índices de DCVs e, consequentemente, de suas complicações e mortalidades, sobretudo pelo de fatores sociais modificáveis apresentarem tamanha importância epidemiológica para o seu desenvolvimento.

Cabe lançar luz à importância de estudos futuros realizados por profissionais de saúde, avaliando a real presença dos FR, pois esta pode estar subestimada por ser auto-referida pela população que, se desconhece sobre as condições patológicas que possui, não as informa, gerando grande impacto tanto pessoal quanto comunitário, posto que doenças e práticas prejudiciais podem ser perpetuadas entre indivíduos de uma mesma família ou comunidade.

O estudo do autoconhecimento é, neste contexto, de grande valia também para contribuir com a conscientização, que auxilia na promoção do autocuidado e na procura pela saúde, além de ter caráter difundível. A Atenção Primária à Saúde tem em sua definição o objetivo de promoção e prevenção, bem como o empoderamento da população assistida, a partir da expansão de conhecimentos, não só acerca da saúde de maneira específica, mas também de direitos e de fatores relacionados à saúde e à independência social, como educação, saneamento, trabalho, moradia e exposição a fatores de risco. Nesse contexto, a prevenção primária com equipes multidisciplinares é importante instrumento para evitar ou atrasar o desenvolvimento das DCV, conscientizando a população, independentemente de sua região, provendo de conhecimento e educação acerca dos fatores de risco CV.

Todas as medidas que corroborem para $\mathrm{o}$ desenvolvimento humano e social, com foco na melhora dos indicadores socioeconômicos devem ser priorizadas pelas gestões públicas, a fim de melhorar os determinantes sociais de saúde e, assim, a sobrevida e a qualidade de vida das populações.

Devemserestimuladas, pelos órgãos responsáveis, eventos com atividades que possam promoção de saúde e aprimorar estratégias de combate aos fatores de risco, desempenhando mais adequadamente a política de prevenção primária em saúde, que compõe os dois pilares da atenção primária à saúde.

\section{Conclusão}

$\mathrm{Na}$ avaliação do autoconhecimento dos fatores de risco CV dos indivíduos moradores de ambas as regiões, urbana periférica e rural, foi identificado importante desconhecimento de suas condições em ambos os grupos. Apesar dos FR CV terem sido mais evidentes na ZR, o relato da presença no histórico pessoal, além de sintomas sugestivos do sistema $\mathrm{CV}$, foram mais proeminentes na ZUP, com a observação de se tratar de um grupo mais jovem. Medidas de prevenção primária devem ser constantemente estimuladas, independentemente da região de moradia, no sentido da conscientização em relação aos fatores de risco de $\mathrm{CV}$.

\section{Referências}

1: World Health Organization [internet]. Cardiovascular disease. [acesso em 29/01/2020] Disponível em http://origin.who.int/cardiovascular diseases/ en/

2: Arruda GO, Santos AL, Teston EF, Cecilio HPM, Radovanovic CAT, Marcon SS. Associação entre autopercepção de saúde e características sociodemográficas com doenças cardiovasculares em indivíduos adultos. Rev Esc Enferm USP 2015 Fev; 49(1): 61-68.

3: Siqueira ASE, de Siqueira-Filho AG, Land MGP. Análise do Impacto Econômico das Doenças Cardiovasculares nos Últimos Cinco Anos no Brasil. Arq Bras Cardiol 2017 Jul; 109 (1): 39-46.

4: Faludi AA, Izar MCO, Saraiva JFK, Chacra APM, Bianco HT, Afiune NA, et al. Atualização da Diretriz Brasileira de Dislipidemias e Prevenção da Aterosclerose. Arq Bras Cardiol 2017 Ago; 109(2 Suppl 1): 1-76.

5: Organização das Nações Unidas [internet]. Comissão da OMS pede ação urgente contra doenças crônicas não transmissíveis. [Acesso em 29/01/2020] Disponível em: https://nacoesunidas.org/comissao-da-oms-pede-acaourgente-contra-doencas-cronicas-nao-transmissiveis/

6: Projeto Brasil Saúde Amanhã [internet]. Condicionantes Sociais e Econômicos da Saúde. [Acesso em 29/01/2020] Disponível em: https:// saudeamanha.fiocruz.br/condicionantes-sociais-e-economicos-da-saude/\#. XjIY-2hKjIU

7: de Andrade L, Zanini V, Batilana AP, de Carvalho EC, Pietrobon R, Nihei $\mathrm{OK}$, et al. Regional Disparities in Mortality after Ischemic Heart Disease in a Brazilian State from 2006 to 2010. PLoS One 2013; 8(3): e59363.

8: Rodrigues TC, Lima MHM, Nozawa MR. O controle do diabetes melittus em usuários de Unidade Básica de Saúde, Campinas, SP. Ciênc Cuidado Saúde 2006; 5(1):41-9

9: Magnabosco P, Cavalari TE, Meirelles de OE, Felipe EA, Freitas D, Marchi-Alves LM. Análise comparativa da não adesão ao tratamento medicamentoso da hipertensão arterial sistêmica em população urbana e rural. Rev Latino-Am Enfermagem 2015 Jan-Fev; 23(1):20-7. 
10: Precoma DB, Oliveira GMM, Simão AF, Dutra OP, Coelho OR, Izar MCO, et al. Atualização da Diretriz de Prevenção Cardiovascular da Sociedade Brasileira de Cardiologia - 2019. Arq Bras Cardiol 2019; 113(4):787-891.

11: Havranek EP, Mujahid MS, Barr DA, Blair IV, Cohen MS, CruzFlores S, et al; American Heart Association Council on Quality of Care and Outcomes Research, Council on Epidemiology and Prevention, Council on Cardiovascular and Stroke Nursing, Council on Lifestyle and Cardiometabolic Health, and Stroke Council. Social determinants of risk and outcomes for cardiovascular disease: a scientific statement from the American Heart Association. Circulation 2015 Set;132(9):873-98

12: Soares GP, Klein CH, Souza e Silva NA. Evolution of mortality from diseases of the circulatory system and of gross domestic product per capita in the Rio de Janeiro State Municipalities. Arq Bras Cardiol 2013 Mar;100(2):147-56

13: Nunes KVR, Ignotti E, Hacon SS. Circulatory disease mortality rates in the elderly and exposure to PM2.5 generated by biomass burning in the Brazilian Amazon in 2005. Cad Saúde Pública 2013 Mar;29(3):589-98.

14: Massa KHC, Duarte YAO, Filho ADPC. Análise da prevalência de doenças cardiovasculares e fatores associados em idosos, 2000-2010. Ciênc. Saúde Colet. 2019 Jan 24 (1): 105-114.

15: Wilson PW, D’Agostino RB, Levy D, Belanger AM, Silbershatz H, Kannel WB. Prediction of coronary heart disease using risk factor categories. Circulation 1998 Mai; 97(18): 1837-47.

16: Eckel RH. Obesity and heart disease: a statement for healthcare professionals from the Nutrition Committee, American Heart Association. Circulation 1997 Nov; 96(9): 3248-50.

17: NHLBI Obesity Education Initiative Expert Panel on the Identification, Evaluation, and Treatment of Obesity in Adults (US). Clinical Guidelines on the Identification, Evaluation, and Treatment of Overweight and Obesity in Adults: The Evidence Report. Bethesda (MD): National Heart, Lung, and Blood Institute; 1998 Sep. Disponível em: https://www.ncbi.nlm.nih.gov/ books/NBK2003/

18: Fletcher GF, Balady G, Blair SN, Blumenthal J, Caspersen C, Chaitman $\mathrm{B}$, et al. Statement on exercise: benefits and recommendations for physical activity programs for all Americans: a statement for health professionals by the Committee on Exercise and Cardiac Rehabilitation of the Council on Clinical Cardiology, American Heart Association. Circulation 1996 Ago; 94(4):857-62

19: Loures DL, Sant'Anna I, Baldotto CSR, Sousa EB de, Nóbrega ACL da. Estresse Mental e Sistema Cardiovascular. Arq Bras Cardiol 2002 Mai; 78(5): 525-530.

20: De Azevedo BMB, Bergamo FPMS, Margarete ZL, Galvão CCL. Tendências das desigualdades sociais e demográficas na prevalência de doenças crônicas no Brasil, PNAD: 2003- 2008. Ciênc. Saúde Colet. 2011 Set 16(9): 3755-3768.

21: Duncan BB, Schmidt MI, Polanczyk CA, Meneghel S. Altas taxas de mortalidade por doenças não transmissíveis em populações brasileiras - uma comparação internacional. Rev Ass Médica Brasileira 1992 Jul; 38: 138-44

22: Piepoli MF, Hoes AW, Agewall S, Albus C, Brotons C, Catapano AL, et al. 2016 European Guidelines on cardiovascular disease prevention in clinical practice. The Sixth Joint Task Force of the European Society of Cardiology and Other Societies on Cardiovascular Disease Prevention in Clinical Practice (constituted by representatives of 10 societies and by invited experts). Eur Heart J 2016 Ago; 37(29):2315-81.

23: Albert MA, Glynn RJ, Buring J, Ridker PM. Impact of traditional and novel risk factors on the relationship between socioeconomic status and incident cardiovascular events. Circulation 2006 Dez;114(24):2619-26.

24: Alter DA, Franklin B, Ko DT, Austin PC, Lee DS, Oh PI, et al. Socioeconomic status, functional recovery, and long-term mortality among patients surviving acute myocardial infarction. PLoS One 2013 Jun;8(6):e65130.

25: Rajagopalan S, Al-Kindi S, Brook R. Air pollution and cardiovascular disease. J Am Coll Cardiol 2018 Out; 72(17):2054-70.

26: Mesquita, CT. Relação entre Fatores Sociais e Doenças Cardiovasculares. Int J Cardiovasc Sci 2018 Mar-Abr;31(2)87-89.
27: da Silva EF, Paniz VMV, Laste G, Torres ILS. Prevalência de morbidades e sintomas em idosos: um estudo comparativo entre zonas rural e urbana. Ciênc Saúde Coletiva 2013 Abr; 18( 4 ): 1029-1040.

28: Lima-Costa MF, Peixoto SV, Firmo JOA, Uchoa E. Validade do diabetes auto-referido e seus determinantes: evidências do projeto Bambuí. Rev Saúde Pública 2007 Dez; 41(6): 947-953.

29: Souza A, Medeiros AR, Souza AC, Wink M, Siqueira IR, Ferreira MBC, el at. Avaliação do impacto da exposição a agrotóxicos sobre a saúde de população rural. Vale do Taquari (RS, Brasil). Ciênc Saúde Coletiva 2011 Ago; 16(8):3519-3528. 